Proceedings of the ASME 2019 38th International Conference on Ocean, Offshore and Arctic Engineering

OMAE2019

June 9-14, 2019, Glasgow, Scotland, UK

OMAE2019-96716

\title{
RESEARCH ON CHARACTERISTICS OF REMOVING PARTICLES IN SHIP EXHAUST GAS BY CHARGED DROPLET
}

\author{
Lei Jiao \\ Zhaohui Qian ${ }^{1}$ \\ Zhejiang University \\ Zhejiang University \\ Zhoushan, Zhejiang, P.R.China \\ Zhoushan, Zhejiang, P.R.China \\ Pengfei Chen \\ Peilin Zhou \\ Zhejiang University \\ Zhejiang University \\ Zhoushan, Zhejiang, P.R.China \\ Zhoushan, Zhejiang, P.R.China \\ University of Strathclyde \\ Glasgow, UK
}

Dekai Huang

Zhejiang University

Zhoushan, Zhejiang, P.R.China

Lida Meng

Zhejiang University

Zhoushan, Zhejiang, P.R.China

\section{ABSTRACT}

A traditional water scrubber is able to remove particles with a size over $200 \mu \mathrm{m}$ in ship engine exhaust effectively. However, as the size of the particles decreases, the removal efficiency of the particles is gradually reduced, especially when the particle size is less than $50 \mu \mathrm{m}$, the method amolst has little effect. This paper presents a study charging particles in exhaust gas and water droplets to improve water scrubber's efficiency in removing fine particles. The charging of the particles is mainly achieved through corona discharge, while the water droplets are charged by passing the high-voltage electricity to the nozzle. However, the feasibility and economics of these two methods have not been verified in other researches, so they are numerically simulated by Comsol Multiphysics software in this paper. The simulation results shows that both particles and droplets can be charged steadily by the two methods. The numerical simulation results also indicates that the removal efficiency of particles in ship exhaust gas can be greatly improved by adding charges to droplets and particles at the same time. And a line chart of particle capture efficiency map under different particle size and change of droplet is obtained.

Keywords: Particles, Charged droplet, Comsol Multiphysics, Coulomb force

\section{INTRODUCTION}

With the development of the shipping industry, ship exhaust pollution including sulfides, nitrogen oxides and carbonaceous particles has also received increasing attention. .Nowadays, the sulfides in ship's exhaust gas are mainly removed by gypsum flue gas desulfurization method, rotary spray drying desulfurization method and seawater desulfurization method. For nitrogen oxides, they are mainly removed by non-catalytic reduction method (SNCR) and selective catalytic reduction method (SCR)[1]. The desulfurization and denitration technologies for ship exhaust gas are relatively mature, which can reach more than $80 \%$ of removal efficiency of SOx and NOx in ship[1]. Now, for inhalable particles, it is mainly removed by a wet scrubber, that is, the washing liquid is turned into fine droplets through a nozzle to absorb particles. Through this method the

Accepted author manuscript of the following research paper: Jiao, L., Qian, Z., Huang, D., Zhou, P., Chen, P., \& Meng, L. (2019). Research on characteristics of removing particles in ship exhaust gas by charged droplet. In ASME 2019 38th International Conference on Ocean, Offshore and Arctic Engineering (Vol. 7A). ASME. https://doi.org/10.1115/OMAE2019-96716 


\section{ACCEPTED DRAFT}

removal efficiency of the particles larger than $200 \mu \mathrm{m}$ can be close to $100 \%$, but the removal efficiency of small particles is very low[2]. In the ship's exhaust gas, most of the particles have a particle size below $200 \mu \mathrm{m}$, and the proportion is even more than $90 \%$. Therefore, the efficiency of the removal of particles by conventional wet scrubbers is not guaranteed to meet international standards. When the unremoved particles is discharged into the atmosphere, it will seriously pollute the environment and enter the human body through the respiratory system, which seriously endangers the health of the human body.

The use of charged droplets to remove particles is based on a conventional wet scrubber. By means of corona discharge and passing high voltage on the nozzle, the particles and droplets are respectively charged with opposite charges, and Coulomb force is generated between the two. The force, working with the inertial collision interception and Brownian diffusion in the wet scrubber, greatly increases the adsorption effect of the droplets on the particles and improves the removal efficiency of the particles.

\section{WORKING PRINCIPLES AND THEORETICAL MODEL}

The principles of particles removal by charged droplets are very complicated. Traditional wet scrubbers rely mainly on inertial collision, interception and Brownian diffusion. For the charged droplets, the Coulomb force is added to the particles removal mechanism.

In this paper, the theoretical formula for the adsorption of particles by a single droplet is discussed by the principles of the removal of atmospheric aerosols in lightning weather. The evaporation of the droplets and the thermal gradient in the reaction chamber are omitted for simplicity. The instantaneous rate formula for removing particles is as follows[3]:

$n^{\prime}\left(d_{p}, t\right)=n\left(d_{p}, t\right)\left[\frac{\pi}{4}\left(D+d_{p}\right)^{2} U\right] E$

Where $n^{\prime}\left(d_{p}, t\right)$ is the amount of particles removed in time t, $n\left(d_{p}, t\right)$ is the concentrated number of particles in time $t, D$ is the diameter of droplet, $d$ is the diameter of the particles, $U$ is the rate of exhaust gas, $\mathrm{E}$ is the collision coefficient which is related to various interactions between droplets and particles. The bracketed part of Equation (1) is the volume of the cylinder swept by a droplet, which is the collision cylinder. It can be seen from the above formula that the absorption efficiency of the particles mainly depends on the collision coefficient E. Usually, $\mathrm{E}$ is less than 1, indicating that only part of the particles in the collision cylinder are adsorbed and removed; but $\mathrm{E}$ may also be greater than 1 , indicating that the particles outside the collision column may also be adsorbed and removed[3].
The total collision coefficient $\mathrm{E}$ in Equation 1 can be regarded as the sum of the collision coefficients of various adsorption mechanisms in reaction chamber, mainly related to Brownian diffusion, inertial collision, interception and electrostatic interaction. So the formula for $\mathrm{E}$ can be written as[2]:

$\mathrm{E}=E_{\text {diff }}+E_{\text {int }}+E_{\text {imp }}+E_{\text {ele }}$

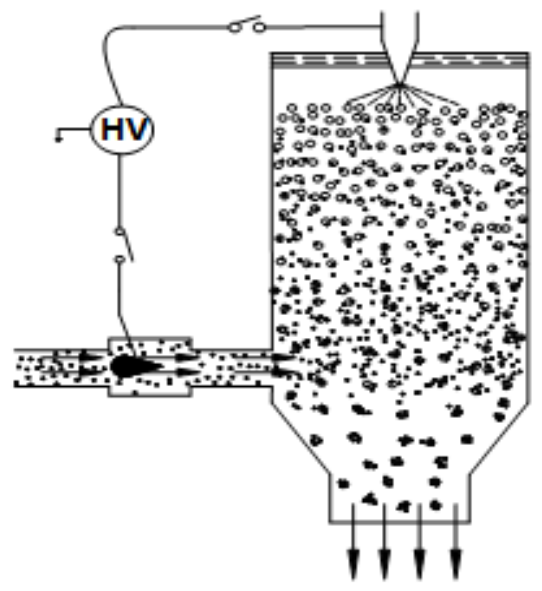

Figure 1. Sketch of particles adsorption device by charged droplets

\subsection{INERTIAL COLLISION}

Inertia collision mainly means that when a gas encounters an obstacle (in this case, a droplet), it will cause a turbulence phenomenon on its surface. For a particle with a large particle size, it will advance along its original motion trajectory, and captured by obstacles due to the large inertial force .[4] The calculation formula of the collision coefficient of inertial collision is as follows[5][6][7]:

a. Slinn Equation

$\mathrm{St}=\frac{C_{C} \rho_{p} d_{p}^{2} \gamma C}{18 \mu d_{D}}, \mathrm{~S} t^{*}=\frac{\frac{1}{2}+1 / 12 \ln (1+R e)}{1+\ln (1+R e)}$

b. Licht Equation

$E_{\text {imp }}=\left[\frac{S t}{S t+0.36}\right]^{2}$

c. Kim Equation

$E_{\text {imp }}=3.4 S t^{9 / 5}, S t \leq 0.5$

$E_{\text {imp }}=1, S t>0.5$

It can be seen from the above formula that the efficiency of inertial collision is mainly related to the diameter of the particles. When the size of the particles is larger, the effect is more obvious. 


\section{ACCEPTED DRAFT}

\subsection{INTERCEPTION}

Intercepting mainly means that when the particles flow with the gas, if the distance between the droplet and the streamline is smaller than the radius of the particles, the particles will be adsorbed by the droplet.[8] The collision coefficient of the interception is calculated as follows[5][7]:

a. Slinn Equation

$E_{\text {int }}=4 H\left[\omega^{-1}+\left(1+2 R e^{\frac{1}{2}}\right) H\right]$

$\omega=\frac{\mu_{\omega}}{\mu}, \mathrm{H}=\frac{d_{p}}{D}$

b. Jung and Lee Equation

$$
\begin{aligned}
& E_{\text {int }}=\frac{(1-\alpha)}{(J+\omega K)}\left[\left(\frac{H}{1+H}\right)+\frac{1}{2}\left(\frac{H}{1+H}\right)^{2}(3 \omega+4)\right] \\
& \mathrm{J}=1-\frac{6}{5} \alpha^{\frac{1}{3}}+\frac{1}{5} \alpha^{2}, \omega=\frac{\mu_{\omega}}{\mu} \\
& \mathrm{K}=1-\frac{9}{5} \alpha^{\frac{1}{3}}+\alpha+\frac{1}{5} \alpha^{2}, H=\frac{d_{p}}{D}
\end{aligned}
$$

It can be seen from the above equation that the collision coefficient of the interception is mainly related to the size of the particles and droplets.

\subsection{BROWNIAN DIFFUSION}

Brownian diffusion in this paper mainly refers to the phenomenon that particles collide with particles in the reaction chamber to change their trajectories. This phenomenon increases the collision probability of droplets and particles, thereby increases its adsorption efficiency. The calculation formula of the collision coefficient of Brownian diffusion is as follows[5]:

$$
\begin{aligned}
& E_{\text {diff }}=\frac{4}{\operatorname{ReSc}}\left[1+-0.4 \operatorname{Re}^{\frac{1}{2}} S c^{\frac{1}{3}}+0.16 \operatorname{Re}^{\frac{1}{2}} S c^{\frac{1}{2}}\right] \\
& \mathrm{Sc}=\frac{\mu}{\rho D_{\text {diff }}}, \quad D_{\text {diff }}=\frac{K_{B} C_{C} T_{a S}}{3 \pi \mu d_{p}}
\end{aligned}
$$

\subsection{ELECTROSTATIC INTERACTION}

By charging the corona and applying high voltage to the nozzle, the particles and the droplets can be charged relatively. When only particles or only droplets are charged, a mirror charge force will be generated between the two. If the particles and droplets carry different charges, a Coulomb force is generated between the two, which is an electrostatic attraction. If the particles and droplets carry the same charge, a Coulomb force is generated between them, and this is an electrostatic repulsion. All three electrostatic forces enhance the adsorption of droplets on the particles, but the effect is most pronounced when both are charged. The formula for calculating the electrostatic interaction collision coefficient is as follows[9]:

$E_{\text {ele }}=\frac{16 K_{C} C_{C} q q_{p}}{3 \pi \mu U D^{2} d_{p}}$

The charge of the droplet must not be higher than its Rayleigh limit $\left(\mathrm{q}_{\mathrm{R}}\right)$, because once this limit is exceeded, the droplets will no longer stabilize and break into small droplets. For droplets with a dielectric constant of $\varepsilon$, a diameter of $\mathrm{D}$, and a surface tension of $\Gamma_{\omega}$, the Rayleigh limit is calculated as follows[10]:

$q_{R}=2 \pi \sqrt{2 \varepsilon \Gamma_{\omega} D^{3}}$

The charging of the particles can be achieved by means of corona discharge. The particles themselves carry a small amount of charge, but by corona discharge, the charge of the particles can be increased to about 10 times. The initial charge amount of the particles is calculated as follows[11]:

$\left|q_{p}\right|=A d_{p}{ }^{B} \cdot e$

Where $\mathrm{A}=36.8, \mathrm{~B}=1.17$, the measurement unit of $\mathrm{d}_{\mathrm{p}}$ is micrometer.

The realization of the method mainly includes the feasibility of charging the particles by corona discharge, the feasibility of charging the nozzle by applying high voltage to the nozzle and the high adsorption rate of the charged particles to the charged particles. The content of the following paper is also mainly divided into the above three modules one by one to explain, and through numerical simulation to verify.

\section{PARTICLES CHARGING MODULE}

\subsection{THEORETICAL BASIS FOR CHARGED PARTICLES}

The charging method of the particles is corona discharge, that is, the electric charge is loaded into the particles in the electrically neutral gas by means of corona discharge. Many of these gas movement phenomena can be studied, including electric field and charge distribution, fluid motion and heat transfer[12].

The mechanism of pulsed corona particle flow can be roughly understood by Figure 2. When the particles is close to the corona discharge region, since the particles is charged due to the presence of a strong electric field at the high-end corona electrode, the operating voltage range of the corona discharge is substantially between the corona start voltage and the air gap breakdown voltage[13]. The choice of corona electrodes depends on multiple factors, including electrode material, device shape, 
gas generation, and others.

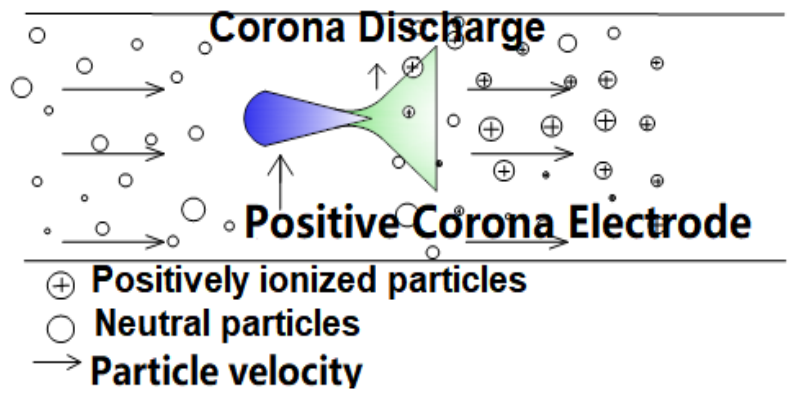

Figure 2. Schematic diagram

\subsection{NUMERICAL MODEL OF CHARGED PARTICLES}

\subsubsection{MATHEMATICAL MODEL}

The particle charging mode can be described by the formula (12).

$\nabla^{2} V=-\frac{\rho}{\epsilon_{0}}$

Where $\mathrm{V}$ is determined by the Poisson equation, $\rho$ is the space charge density, and $\varepsilon_{0}$ is the free space dielectric constant. The potential is determined by the electric field strength:

$\mathrm{E}=-\nabla \mathrm{V}$

The total current is mainly composed of three parts, namely, electrical conduction (gas ion current under the action of electric field), convection (charge of gas flow) and diffusion[12].

$\mathrm{J}=\mu_{E} E \rho+U \rho-D \nabla \rho$

Where $\mu_{\mathrm{E}}$ is the mobility of the charged particles in the electric field, $\mathbf{U}$ is the velocity vecyor of gas, and D is the ion diffusion coefficient. According to current continuity, current density can be described as[12]:

$\nabla \cdot \mathrm{J}=0$

The governing equations of fluid dynamics are mainly N-S equations and continuity equations for steady state incompressible gases[12].

$\rho_{\text {air }} U \cdot \nabla U=-\nabla p+\mu \nabla^{2} U-\rho \nabla V$

$\nabla \cdot \mathrm{U}=0$

Where $\rho_{\text {air }}$ is the gas density, $\mathrm{p}$ is the gas pressure, and $\mu$ is the gas dynamic viscosity.

\subsubsection{NUMERICAL SIMULATION RESULTS OF PARTICLES CHARGING}

Turbulent flow and electrostatic (AC/DC) modules in COMSOL Multiphysics software were used to calculate the simulation of particles charging. Figure 3 shows the model of the module, where a is the wall, b is the gas inlet, c is the gas outlet, and $\mathrm{d}$ is the corona electrode. Set a constant voltage on the surface of the corona electrode, set 0 voltage in the upper and lower closets.

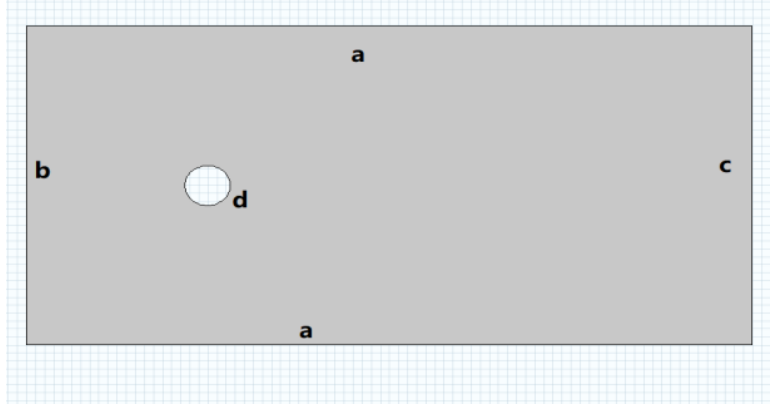

Figure 3. Particle charging module model diagram

Finally, the results of the numerical simulation can be obtained by solving the solver. The airflow velocity diagram is shown in Figure 4, which are generated with a corona electrode to channel walls potential difference of $8 \mathrm{kV}$. The ionic current generated by corona discharge may interfere with the movement of fluids and particles in the flow channel so that the particles cannot reach the reaction chamber. In order to observe the trajectory of the particles clearly, at the time of post-processing, the band-quality particle tracking was calculated. In the figure. Red dots in the figure are particles, and black lines are particle motion tracks. It can be found that almost all of the particles can pass through the corona discharge zone and enter the reaction chamber. However, it can be seen from the simulation results that the corona electrode will intercept some of the particles during the working process, so it should be cleaned from time to time.

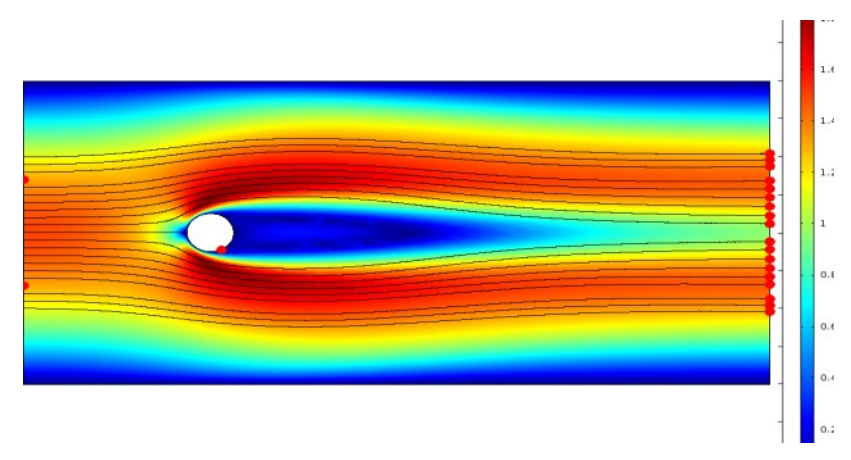

Figure 4. Airflow velocity diagram

\section{DROPLATS CHARGING MODULE}

\subsection{THEORETICAL BASIS OF DROPLETS CHARGING}

The droplets charging method mainly adopts the generation 
method of Taylor cone, that is, the way of adding high voltage to the nozzle. The main principle is the process of atomizing the liquid by applying an electric field force to the flow of water flowing out of the capillary nozzle. Because of the force of gravity, electric force, etc., the jet of water is roughly conical, and there is a fine water flow at the tip of the vertebral body, and finally the water flow is dispersed into fine droplets. The maximum charge of a droplet produced by

Taylor cone is typically half the Rayleigh limit, and the Rayleigh limit is the maximum amount of charge that can be applied to the droplet, at which point the charge produces a repulsive force equal to the surface tension. The charged droplets device usually consists of a capillary nozzle, a grounding electrode and a syringe pump maintained at a high potential.[14]

Generating charged droplets by generating Taylor cone has many advantages over other conventional charged droplets systems that are charged by induction:

1. The droplet size distribution is usually consistent and the standard deviation is small;

2. Droplet size is usually smaller than conventional charged droplets and can even be less than $1 \mu \mathrm{m}$.

3. The droplets carry more charges, which reduces the condensation and solidification of the droplets.

4. The movement of charged droplets can be better controlled.

\subsection{NUMERICAL MODEL OF DROPLETS CHARGING}

\subsubsection{MATHEMATICAL MODEL OF DROPLETS CHARGING}

Similar to the charging of particles, the governing equation of the electric field can be written as:

$$
\nabla^{2} \varphi=-\frac{Q}{\varepsilon_{0}}
$$

Where $\varphi$ is the potential, Q is the space charge density, and $\varepsilon_{0}$ is the dielectric constant in vacuum.

In order to describe the electrohydrodynamic equation of the electric current, the dynamic equation of the conductor can be analyzed first, and the electric field force is calculated as the surface force, and the electric field force can be treated as the surface tension:

$\rho \frac{\partial u}{\partial t}+\nabla \cdot(\rho u u)=\rho g-\nabla \cdot p+\mu \nabla^{2} u+F_{S T}+\frac{1}{2 \varepsilon_{0}} \sigma^{2} e_{n}$

Where $u$ is the velocity of fluid motion, $\rho$ is the density of the fluid, $t$ is the time, $F_{\text {ST }}$ is the surface tension at the interface of the two phases, $\mathrm{g}$ is the acceleration coefficient of gravity, $\mathrm{P}$ is the pressure, and $\mu$ is the viscosity of the fluid, $\varepsilon_{0}$ is the permittivity of vacuum, $\sigma$ is the surface charge density . Here, the electric field force of the conductor surface is equivalent to the surface tension, and the surface force treatment is adopted. The electric field force acting on the conductor surface breaks through the surface tension to form a jet.

For the dielectric, unlike the conductor, the electric field inside the conductor is 0 , but the electric field inside the dielectric is not 0 , so the electric field force can be regarded as a volume force, and the external force is added to the N-S equation:

$\rho \frac{\partial u}{\partial t}+\nabla \cdot(\rho u u)=\rho g+\mu \nabla^{2} u+F_{S T}+f$

The above equation is the electrohydrodynamic equation[15].

\subsubsection{NUMERICAL SIMULATION RESULTS OF DROPLETS CHARGING}

In the calculation of droplets charging simulation, this paper uses the binomial-phase field and electrostatic (AC/DC) module in the Comsol Multiphysics software. Figure 5 shows the module calculation model. Where A is the fluid in the nozzle needle, the material is set to water; B is the nozzle needle tube wall, the material is set to stainless steel. Table 1 shows the dimensions and boundary conditions of each boundary of the calculation model. [16].

In order to make the mesh more precise and reduce the calculation time, the B area, that is, the nozzle needle tube wall is not included in the calculation area, and divide the grid of the A-zone and the C-zone more closely, the grid in other parts of the $\mathrm{C}$ area is more rough.

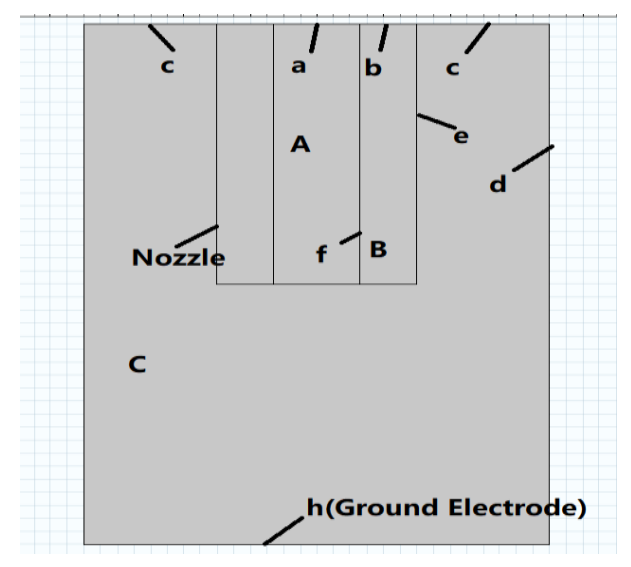

Figure 5.Droplets charging module calculation model diagram

Table 1. Dimensions and boundary conditions of each boundary of droplets charging module 


\begin{tabular}{|c|c|c|c|}
\hline Boundary & $\begin{array}{c}\text { Fluid } \\
\text { mechanics }\end{array}$ & $\begin{array}{l}\text { Electrostatic } \\
\text { field }\end{array}$ & Size $(\mathrm{dm})$ \\
\hline $\mathrm{a}$ & $\begin{array}{c}\text { Inlet } \\
\text { pressure, } \\
\mathrm{p}=130 \mathrm{pa}\end{array}$ & Potential V & 0.26 \\
\hline $\mathrm{b}$ & Velocity, u $=0$ & Potential V & 0.17 \\
\hline $\mathrm{c}$ & $\begin{array}{c}\text { Open } \\
\text { boundary, } \\
p=0\end{array}$ & $\mathrm{nD}=0$ & 0.4 \\
\hline $\mathrm{d}$ & $\begin{array}{c}\text { Open } \\
\text { boundary, } \\
p=0\end{array}$ & $\mathrm{nD}=0$ & 2 \\
\hline e & $\begin{array}{c}\text { No slip, } \\
\text { velocity, } \mathrm{u}=0\end{array}$ & Potential V & 1 \\
\hline $\mathrm{f}$ & $\begin{array}{c}\text { No slip, } \\
\text { velocity, } \mathrm{u}=0\end{array}$ & Potential V & 1 \\
\hline $\mathrm{g}$ & $\begin{array}{l}\text { Binomial- } \\
\text { phase } \\
\text { boundary }\end{array}$ & Potential V & 0.6 \\
\hline $\mathrm{h}$ & $\begin{array}{c}\text { Outlet } \\
\text { pressure, } p=0\end{array}$ & Ground $V=0$ & 1.4 \\
\hline
\end{tabular}

When solving the module, the solver is set to a transient solver with a fairly tolerance set to 0.001 . The solution results are as follows:

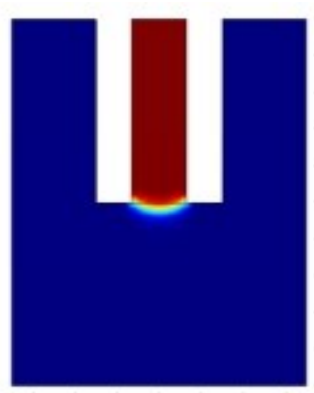

(a).t $=0.001 \mathrm{~s}$

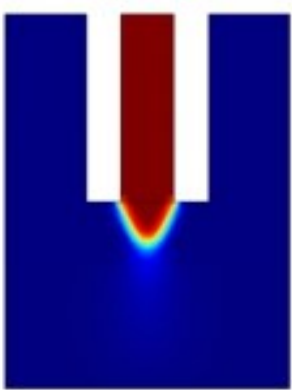

(b).t $=0.0075 \mathrm{~s}$

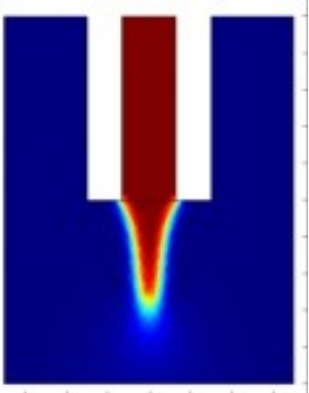

(c).t $=0.009 \mathrm{~s}$

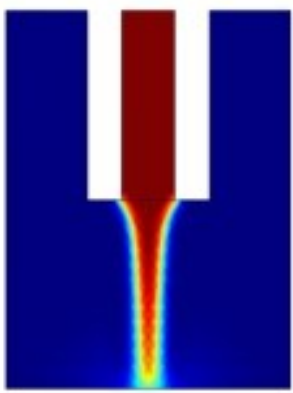

(d).t $=0.0095 \mathrm{~s}$
Figure 6. Morphological change of Taylor cone with time at $5000 \mathrm{~V}$
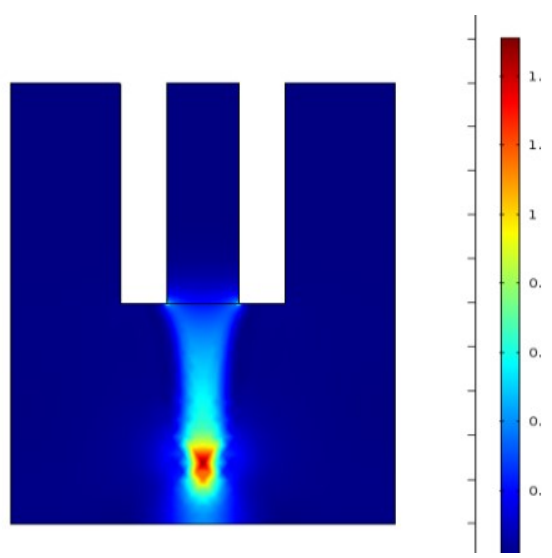

Figure 7. Charge density distribution of Taylor cone surface at $5000 \mathrm{~V}$

It can be seen form Figure 7 that the charge is mainly concentrated at the tip end portion of the fluid. Because of this, the high surface charge density causes the electric field force at the fluid tip to increase, forming a phenomenon of Taylor cone.

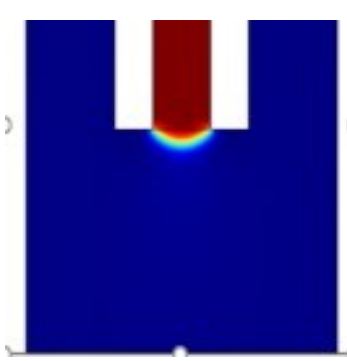

(a). Taylor cone at $3.8 \mathrm{KV}$

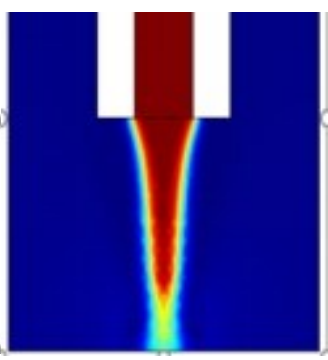

(b). Taylor cone at $4 \mathrm{KV}$ 


\section{ACCEPTED DRAFT}

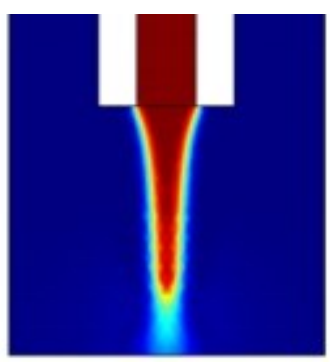

(c). Taylor cone at $4.5 \mathrm{KV}$

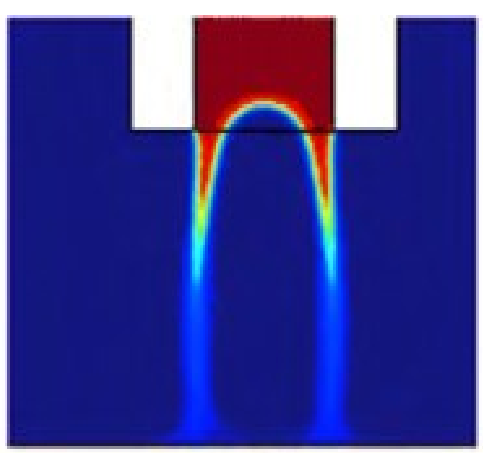

(e) . Taylor cone at $5.5 \mathrm{KV}$

Figure 8. Taylor cone at different voltages

It can be seen from Figure 8 that when the voltage is only $3.8 \mathrm{KV}$, the electric field force is too small to overcome the surface tension of the fluid, so that a Taylor cone cannot be formed. When the voltage is $4 \mathrm{KV}$ or more, Taylor cone can be formed, and when the voltage reaches $5.5 \mathrm{KV}$, a plurality of Taylor cone jets are formed, the jet is unstable, and stable particle removal work cannot be performed.

Therefore, it can be concluded that when a certain range of high-voltage electricity is applied to the nozzle, the nozzle will emit a stable Taylor cone, which can be used to adsorb particles in the ship's exhaust gas.

Table 2. Relationship between applied voltage and formation of Taylor cone in this model

\begin{tabular}{|c|c|}
\hline Applied voltage(KV) & Taylor cone \\
\hline 3.8 & No Taylor cone \\
\hline $4-5$ & $\begin{array}{c}\text { Form a stable Taylor } \\
\text { cone }\end{array}$ \\
\hline 5.5 & $\begin{array}{c}\text { Form a plurality of } \\
\text { Taylor cone jets, the } \\
\text { jet is unstable }\end{array}$ \\
\hline
\end{tabular}

In order to calculate the electrical energy consumption of the charged droplet module, the total energy of the module at 5 $\mathrm{KV}$ is calculated over time during post-processing, as shown in Figure 9.

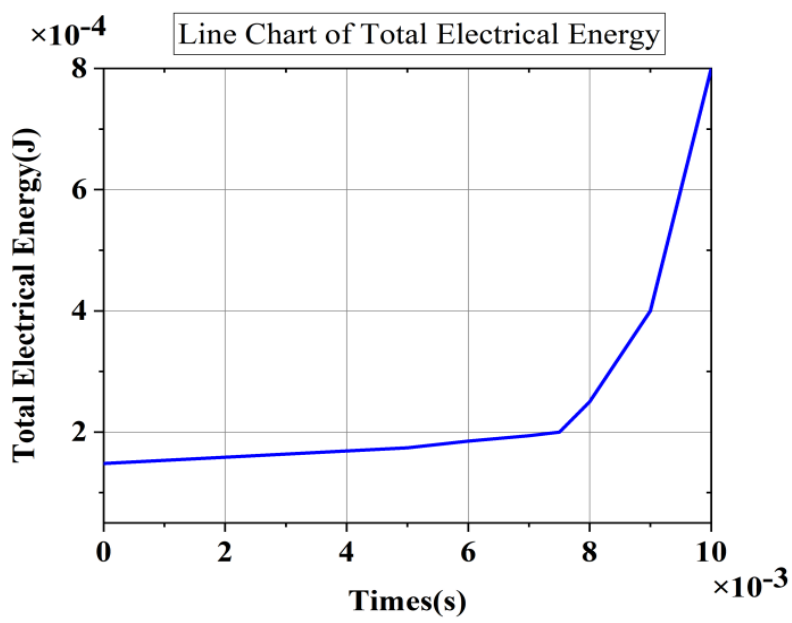

Figure 9. Total energy consumption of charged spray modules

$$
\text { at } 5 \mathrm{KV}
$$

It can be seen from Figure 9 that the power consumption of the module increases steadily after a certain period of time, and the power $\mathrm{P}$ can be estimated to be approximately $0.455 \mathrm{~W}$. It can be seen that the power consumption of the module is not large .

\section{NUMERICAL SIMULATION RESULTS OF THE HIGH EFFICIENCY OF CHARGED DROPLETS TO REMOVE PARTICLES}

\subsection{NUMERICAL SIMULATION RESULTS AND ANALYSIS}

In this part of the numerical simulation, the Electrostatic (AC / DC) module, Particle Tracing for Fluid Flow module, and Turbulent Flow are mainly used. In order to simplify the calculation, the process of trapping particles by droplets is simplified as the blocking effect of static droplets on particles. The Calculation model is shown in Figure 10. 


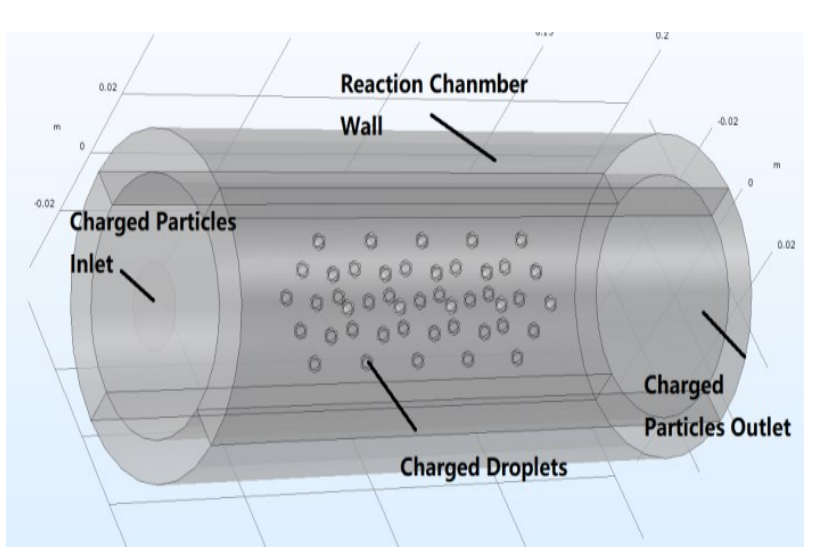

Figure 10. Particle removal efficiency analysis model diagram

In order to better calculate the capturing effect of charged droplets on particulates, the following assumptions are made[17]:

1) The influence of droplets and fine particles on the flow field is ignored during the simulation.

2) It is assumed that the droplets ejected from the nozzles have the same particle size under a certain working condition, and the spatial distribution is relatively uniform.

3) In the simulation, it is assumed that the particles touch the wall surface of the reaction chamber or the surface of the droplet is considered to be trapped, and the orbital tracking of the particles is stopped.

In the parameter setting, the particle size is set to $2 \mu \mathrm{m}$, the charge amount is 2 electrons, and the initial distribution of the inlet particles is evenly distributed, the total number of released particles is 1000 . The size of the droplets is set to $200 \mu \mathrm{m}$, the droplets and fine particles were charged with an opposite charges, and the droplets were set to have a surface charge density of $0.05 \mathrm{C} / \mathrm{m}^{2}$. Since the walls of the reaction chamber are almost all wet walls and the inertia of the particles is relatively small, the wet wall surface around the set is set to the stick boundary, and the surface of the droplet is set as the stick boundary too; the outlet of the reaction chamber is set to the freeze boundary.

To simulate the force of the gas on the particles, a drag force is placed in the computational domain. At the same time, the inertial force, Brownian motion force, gravity and charge force are simulated to simulate the trapping effect of the droplets and the reaction chamber wall on the particles.

When constructing the grid, the free tetrahedral mesh was selected, and the maximum unit size was chosen to be $0.01 \mathrm{~m}$. The model grid diagram is shown in Figure 11:

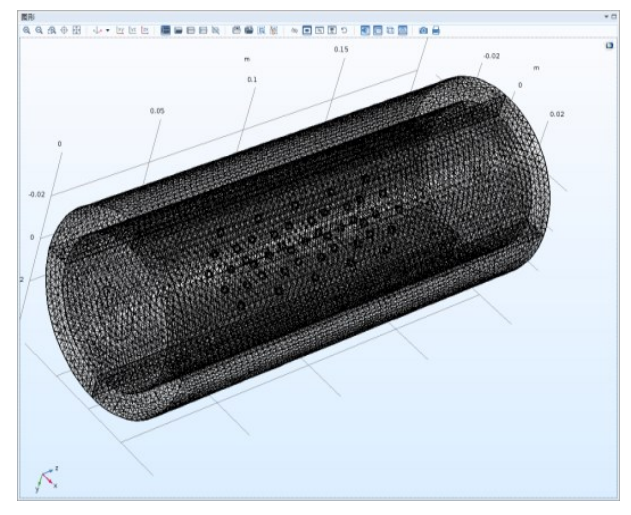

Figure 11. The model grid diagram

This model computes the shape of the particles beam by coupling a time-dependent study step for computing the particle trajectories and a stationary step for computing the electric potential. This algorithm in this simulation consists of the following steps[18]:

1) Compute the particle trajectories, assuming no space charge effects are present, using a time-dependent solver. From these trajectories, compute the space charge density using the Electric Particle Field Interaction node.

2) Compute the electric potential due to the space charge density of the particle beam, using a stationary solver.

3) Use the electric potential calculated in step 2 to compute the perturbed particle trajectories. Recalculate the space charge density using these perturbed trajectories.

4) Repeat steps 2 and 3 until a specified number of iterations has been reached.

Finally, the motion trajectory of the charged particles under the action of charged droplets is shown in the figure below. And the color legend denotes particles' radial displacement over time.

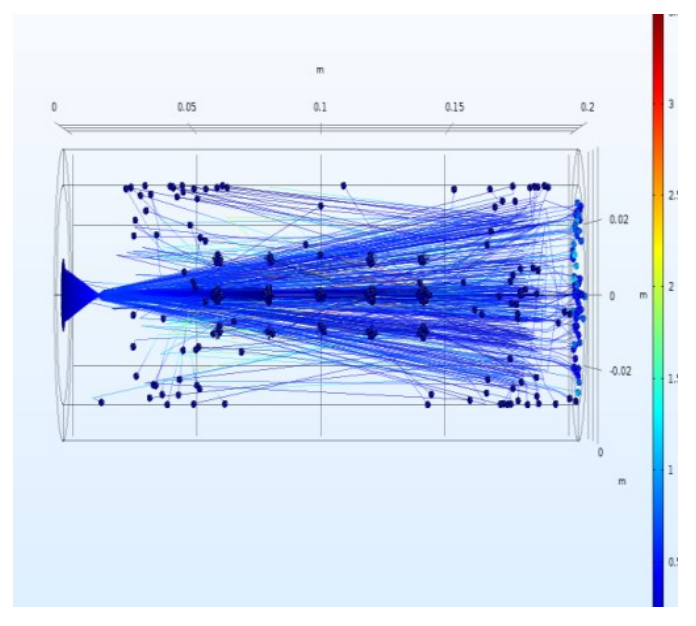


Figure 12. Particle trajectory diagram of charged particles captured by charged droplets

It can be clearly seen from Figure 12 that the particles move toward the droplets and surface of the droplets and chamber wall.

In order to calculate the adsorption efficiency of charged droplets and chamber wall on charged particles, the following figure can be calculated by numerical simulation software.

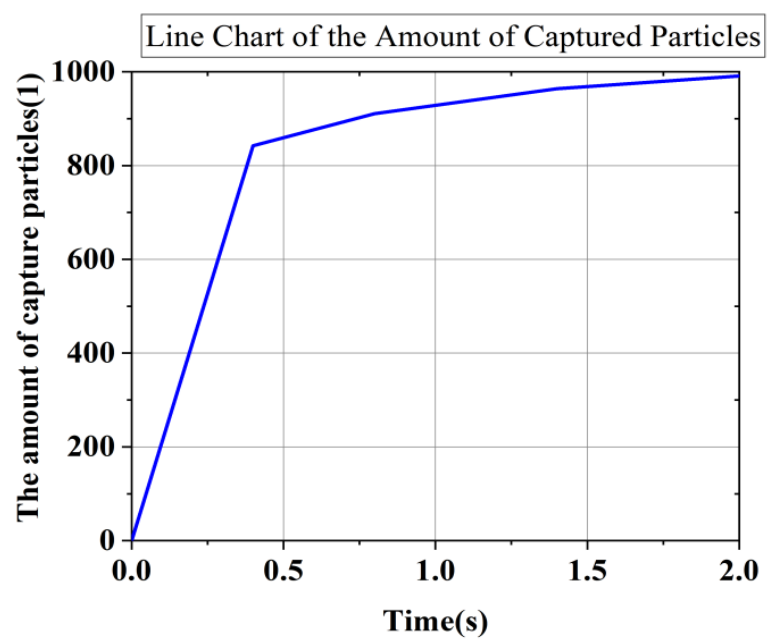

Figure 13. Line chart of the amount of charged particles captured by charged droplets and chamber wall

When calculating the final particle removal efficiency, it can be calculated by the following formula.

$\eta=\frac{C_{2}}{C_{1}}$

Where $C_{1}$ is the initial amount of particles and $C_{2}$ is the number of captured particles. After $2 \mathrm{~s}$ of calculation, the number of particles that eventually captured is 991 , while 1000 particles are released in $2 \mathrm{~s}$. Therefore, by taking the data into equation (20), the particle removal efficiency can be calculated to be about $99.1 \%$.

In order to better illustrate the high efficiency of charged droplets in capturing charged particles, the adsorption rate of the particles is calculated simultaneously when both the particles and the droplets are not charged. The results obtained are as follows

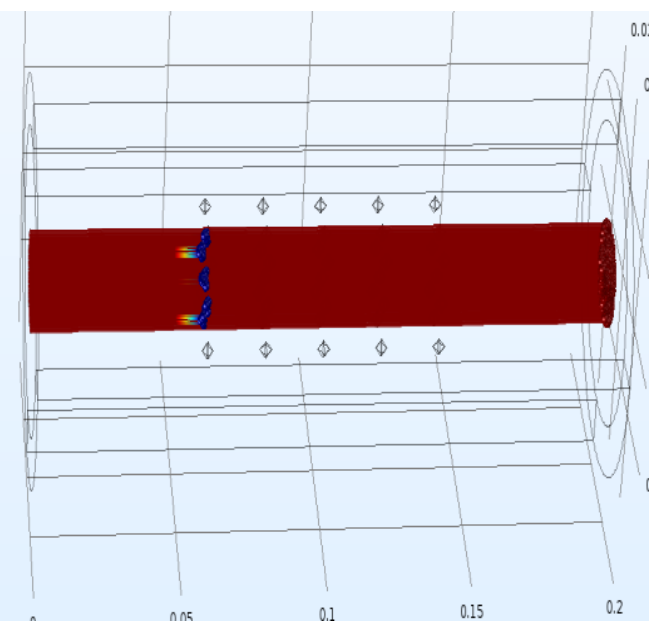

Figure 14. Particle trajectory diagram without electric field force

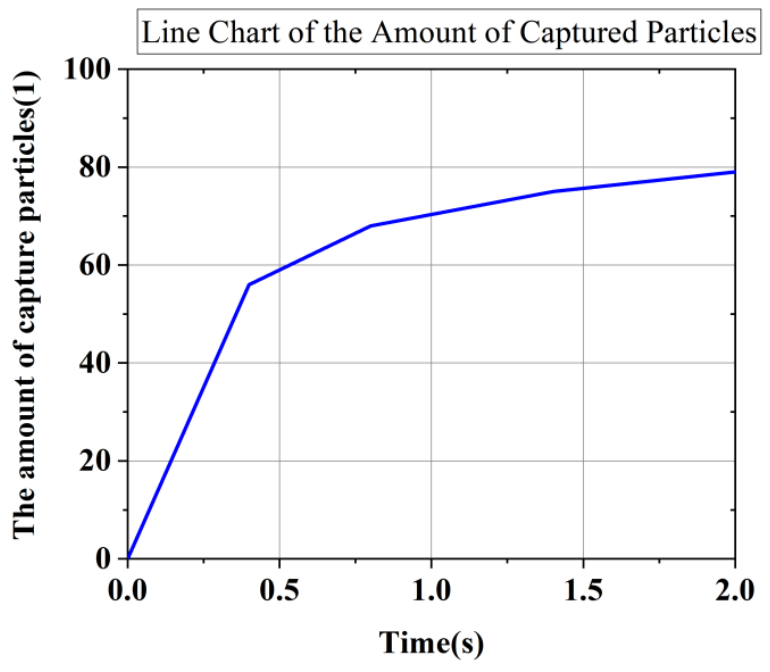

Figure 15. Line chart of the amount of particles captured by droplets and chamber wall

It can be seen from figure 14 that the flow of particles with the gas is almost unaffected by the droplets, and the droplets only capture a small amount of particles. And by formula (20), when the particles and droplets are not charged, the removal efficiency of the particles is only $7.9 \%$.

At the same time, when the particle size and the surface charge density of the droplet are changed, the capture efficiency of each particle size under different charge levels on the surface of the droplet can be obtained, as shown in Figure 16. As can be seen from Figure 16, for particles with a particle size below 1 $\mu \mathrm{m}$, the trapping efficiency is as high as $90 \%$ or more when the droplet charge is only $0.01 \mathrm{C} / \mathrm{m}^{2}$. For particles above $1 \mu \mathrm{m}$, when the droplet charge is the same, the capture efficiency decreases with the increase of the particle size. 


\section{ACCEPTED DRAFT}

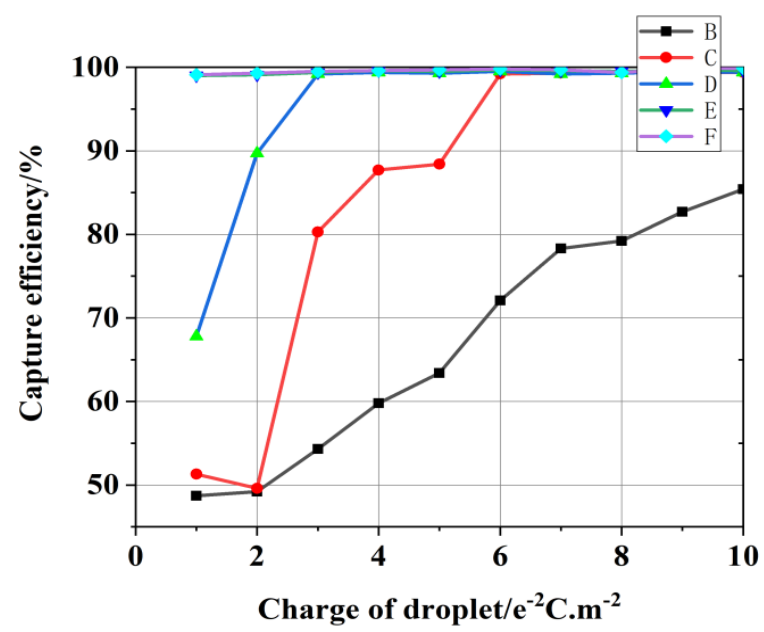

Figure 16. Capturing efficiency of particles of different particle sizes under different charge of droplets

\subsection{VERIFICATION OF ACCURACY OF NUMERICAL SIMULATION METHOD}

In order to verify the accuracy of the numerical simulation method, it can be proved by the difference between the analytical equation of charged particle trajectory and the shape of the charged particle trajectory.

From the force analysis of the dust particles, it is known that after ignoring some minor lateral forces, the particles are all in one plane, so the orbit of the particles is in this plane. For convenience, the vertical plane composed of the $\mathrm{x}$-axis and the $\mathrm{z}$-axis is selected as the plane in which the particle motion trajectory lies. The charged droplets and particles move in opposite directions, the velocity of particles and droplets are $u_{p}$ and $\mathrm{u}_{\mathrm{w}}$ respectively, and $\mathrm{u}_{0}$ is the relative velocity of the two, because the droplet is set here to be stationary, so $\mathrm{u}_{\mathrm{w}}=0, \mathrm{u}_{0}=\mathrm{u}_{\mathrm{p}}$.

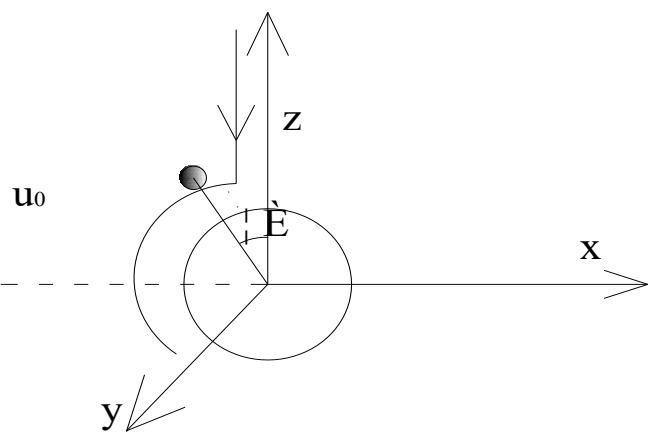

Figure 17.Particle-droplet coordinates

The equation of motion of dust particles in the flow field can be derived from Newton's second law[19]: $m_{p} \frac{d u_{p}}{d t}=\sum F$

The transformation law of space versus time in the coordinate system of Fig. 17 is obtained, and the equation of motion of dust particles is obtained.

$$
\begin{aligned}
& \frac{d^{2} x}{d t^{2}}=-\frac{1}{\tau}\left(u_{p(z)}-u_{x}\right)+\frac{\sum F_{x}}{m_{p}} \\
& \frac{d^{2} z}{d t^{2}}=-\frac{1}{\tau}\left(u_{p(x)}-u_{z}\right)+\frac{\sum F_{z}}{m_{p}}
\end{aligned}
$$

Where, $\mathrm{u}_{\mathrm{p}(\mathrm{x})}=\mathrm{dx} / \mathrm{dt}, \mathrm{u}_{\mathrm{p}(\mathrm{z})}=\mathrm{dz} / \mathrm{dt}$ are the speeds of the dust particles in the $\mathrm{x}$-axis and $\mathrm{z}$-axis directions respectively; $\tau$ is the speed relaxation time, and $F_{x}$ is the viscous resistance. Other forces are in the $\mathrm{x}$-axis direction and $\mathrm{F}_{\mathrm{z}}$ is in the $\mathrm{z}$-axis direction. $\mathrm{u}_{\mathrm{x}}$ and $\mathrm{u}_{\mathrm{z}}$ are the velocity of the airflow around the surface of the droplet. This velocity can be expressed by the velocity of the surface of the droplet. The velocity of the airflow around the droplet can be obtained from the expression of the flow function of the ball[19]:

$u_{z}=\frac{\partial \varphi}{\partial z}=u_{0}-u_{\theta} r_{\omega}^{3} \frac{2 z^{2}-x^{2}}{2\left(x^{2}+z^{2}\right)^{2.5}}$

$u_{x}=\frac{\partial \varphi}{\partial x}=u_{0} \frac{-3 x z r_{\omega}^{3}}{2\left(x^{2}+z^{2}\right)^{2.5}}$

In order to facilitate the numerical solution of the particle motion equation afterwards, it needs to be dimensionless. This paper introduces the dimensionless quantity.

$\mathrm{X}=\frac{\mathrm{x}}{d_{w}}, Z=\frac{z}{d_{w}}, T=\frac{u_{0} t}{d_{w}}$

Considering these dimensionless variables and equation (23) into the equation of motion (22) and sorting them out can be used to obtain a mathematical model of the trajectory of particles when both droplets and particles are charged.

$\frac{d^{2} Z}{d T^{2}}=\frac{-C f}{S_{t k}} \cdot \frac{d Z}{d T}+\frac{C f}{S_{t k}}\left[1-\frac{2 Z^{2}-X^{2}}{16\left(X^{2}+Z^{2}\right)^{2.5}}\right]-\frac{C^{2} \rho_{w} \rho_{p}^{2} d_{p}^{4} \beta_{p} \beta_{w}}{7776 S_{t k}^{2} \mu_{c}^{2} \varepsilon_{0}}$.

$\frac{Z}{\left(X^{2}+Z^{2}\right)^{1.5}}$

$\frac{d^{2} x}{d T^{2}}=\frac{-C f}{S_{t k}} \cdot \frac{d x}{d T}-\frac{3 C f X Z}{16 S_{t k}\left(X^{2}+Z^{2}\right)^{1.5}}-\frac{C^{2} \rho_{w} \rho_{p}^{2} d_{p}^{4} \beta_{p} \beta_{w}}{7776 S_{t k}^{2} \mu_{c}^{2} \varepsilon_{0}} \times \frac{X}{\left(X^{2}+Z^{2}\right)^{1.5}}-$ $\frac{C^{2} d_{p}^{4} \rho_{p}^{2} g}{324 \mu_{c}^{2} d_{w} S_{t k}^{2}}$

Where $\mathrm{C}$ is the Canning correction coefficient, $\mathrm{f}$ is the model motion parameter, $\mathrm{S}_{\mathrm{tk}}$ is the Stokes number, $\varepsilon_{0}$ is the vacuum dielectric constant, $g$ is the gravity acceleration, $\rho_{\mathrm{p}}$ is the density of the particles, $d_{p}$ is the particle size, $\rho_{w}$ is the droplet Density and dp is droplet size.

The mesh refinement by numerical simulation proves shape of the particle trajectory agrees more closely with the analytical 


\section{ACCEPTED DRAFT}

expression of Equation (24)) as the maximum mesh element size is reduced. The particle trajectory is compared to the result of this expression, and the relative error is plotted in Figure18. For all values of the maximum element size, the error shown is computed after three iterations of the solver loop. The relative error is obtained by equation (25).

$\eta=\frac{z-z_{-} a v g}{z_{-} a v g}$

Where $\mathrm{z}$ is the $\mathrm{z}$ coordinate of the particle obtained by equation

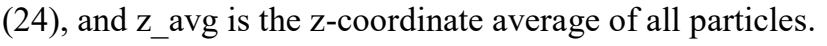

These results show that particle trajectory can be obtained using an iterative solver sequence. This requires much less time and memory than a fully coupled time-dependent study.

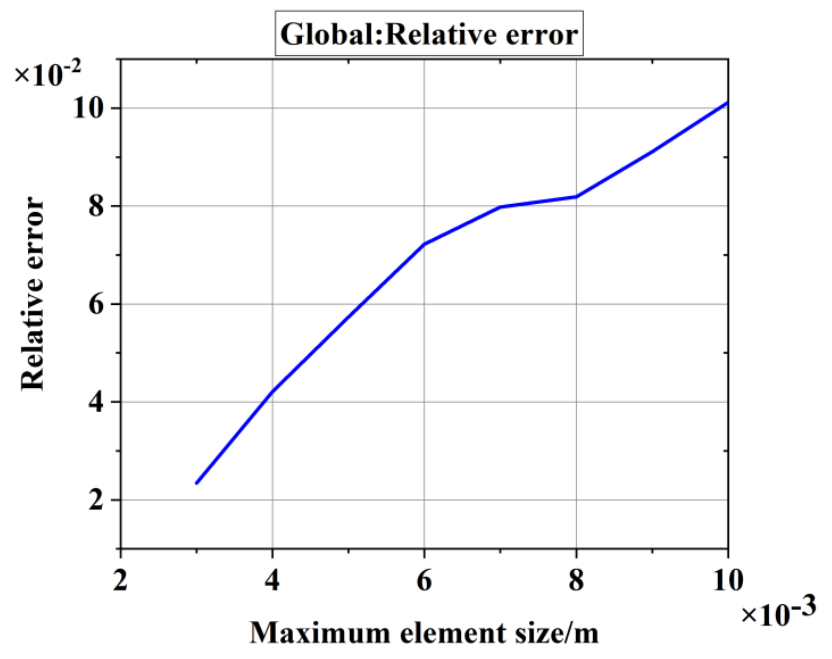

Figure 18. Relative error graph

The accuracy of the solution clearly improves as the mesh element size is reduced, enabling more accurate computation of the particle trajectories. The results of a mesh refinement study indicate that Relative error converges to the expected value as the mesh is refined.

\section{CONCLUSIONS}

Characteristics of removing particles in ship exhaust gas by charged droplet have been researched in this paper. This research can be divided into three modules:particles charging module, droplets charging module and high efficiency of charged droplet to remove particles module.

The charging of particles is achieved by corona discharge method. Theoretical studies have shown that this method can make the particles in the exhaust gas stably charged. At the same time, it is found by numerical simulation that when the voltage of the corona electrode is $8 \mathrm{kV}$, the ion wind generated by corona discharge does not cause great interference to the flow field of the exhaust gas, which proves the feasibility of the method.

Droplet charging is achieved by applying high voltage to the nozzle. The mathematical model of the process can be derived through theoretical analysis. And it is found that the process of droplet charging is the formation process of Taylor cone. By numerical simulation results, it is found that the addition of high voltage to the nozzle can form a stable Taylor cone. Moreover, under the simulated model size, when the applied voltage is $3.8 \mathrm{kV}$, the Taylor cone is not formed. When the applied voltage is $4-5 \mathrm{kV}$, a stable Taylor cone is formed. When the applied voltage is $5.5 \mathrm{kV}$, unstable Taylor cone is formed.. At the same time, in order to verify the economical efficiency of the method, the total energy consumption line chart of the model is plotted when the numerical simulation is postprocessed. It is found that when the applied voltage is $5 \mathrm{kV}$, the power of the model is about $0.455 \mathrm{~W}$ after stable operation.

The high efficiency of charged droplets in capturing charged particles is reflected by the numerical simulation of the adsorption of particles by static array droplets. The numerical simulation results shows that when the droplet size is $200 \mu \mathrm{m}$, the surface charge density is $0.05 \mathrm{C} / \mathrm{m}^{2}$, the particle size is $2 \mu \mathrm{m}$, and the charge is 2 times the electron charge, the capture efficiency of the particles is $99.1 \%$. However, when both particulate matter and droplets are uncharged, the capture efficiency of the particles is only $7.9 \%$. At the same time, when the particle size and the surface charge density of the droplet are changed, numerical simulation results shows that for particles whose charge is 2 times the electron charge with a particle size below $1 \mu \mathrm{m}$, the trapping efficiency is as high as $90 \%$ or more when the droplet charge is only $0.01 \mathrm{C} / \mathrm{m}^{2}$. For particles above $1 \mu \mathrm{m}$, when the droplet charge is the same, the capture efficiency decreases with the increase of the particle size. In order to verify the accuracy and simplicity of iterative solver sequence, the line chart of the relative error of the simulation result and the theoretical mathematical simulation with the maximum grid element size is plotted by numerical simulation post-processing. It is found that the results of a mesh refinement study indicate that Relative error converges to the expected value as the mesh is refined.

\section{ACKNOWLEDGMENTS}

This research is supported by Chinese National key Research and Development Program (No.2017YFC0403800) and Chinese National Natural Science Foundation (NO.51276158).

\section{REFERENCES}

[1] Zhang Huan, Zhong Lu Bin, Chen JingSheng, Zheng YuMing. Research Progress on Ship Tail Gas Desulfurization 


\section{ACCEPTED DRAFT}

and Denitrification Technology [J]. Chemical progress,2016,35(11):3650-3657.

[2] Haibo Zhao, C. G. Zheng. Modeling of Gravitational Wet Scrubbers with Electrostatic Enhancement[J].Chemical Engineering \& Technology 31(12):1824 - 1837 .

[3] L. D'Addio,C. Carotenuto,W. Balachandran,A. Lancia,F. Di

Natale. Experimental analysis on the capture of submicron particles (PM 0.5 ) by wet electrostatic scrubbing[J]. Chemical Engineering Science,2014,106.

[4] Fang RuKang. Environmental Dictionary: Science Press, 2003.

[5] WGN Slinn, Precipitation Scavenging, in: Atmospheric Sciences and Power Production, U.S. Dept. of Energy:Divition of Biomed, Env. Res, 1983(Chapter11).

[6] W.Licht, Air Pollution Control Engineering: Basic Calculation for Particulate Collection, $2^{\text {nd }}$ ed. Maecel Dekker, New York, 1998.

[7] H.T.Kin, C.H.Jung, S.N.Oh, K.W.Lee, Particle removal efficiency of gravitational wet scrubber considering diffution, interception, and impaction, Env.Eng.Sci.18(2)(2001)125-127.

[8] Zhang Yaowen. Experimental Study on Removal of Fine Particles by Electrostatic Spray Cyclone Dust Collector [D].Jiangsu University, 2017.

[9] H.M. Davenport, L.K. Perers, Field studies of atmospheric particulate concentration changes during precipitation, Atmos. Environ. 12(1978)997-1008.

[10] L.Rayleigh, On the equilibrium of liquid conducting masses charged with electricity, Philos. Mag.14(1882)184-195.

[11] M.V. Rodrigues, W.D.J. Marra, R.G. Almeida , J.R. Coury, Measurement of the electrostatic charge in airborne particles -particle charge distribution of different aerosols, Braz.J. Chem.Eng.23(1)(2006) 125-133.

[12] Nel E. Jewell-Larsen, Sergey V. Karpov, Lgor A. Krichtafovitch, Vivi Jayanty, Chih-Peng Hsu, Alexnder V. Mamishev, Modeling of corona-induced electrohydrodynamic flow with COMSOL multiphysics. ESA Annnua Meeting on Electrostatics 2008, Paper EI.

[13]F.W.Peek, Dielectric Phenomen in High Voltage Engineering, New York: McGraw Hill , 1929

[14] Anatol Jaworek, Electrohydrodynamic microencapsulation, Encapsulations Nanotechnilogy in the Agri-Food industry Volume 2, 2016 Pages
[15] Zhang Zhiyuan. Research on EHD micro-scale 3D printing jet mechanism, simulation and experiment [D]. Qingdao Technological University, 2015.

[16] Yang Jianjun, Zhang Zhiyuan, Lan Hongbo, Peng Zilong, Wangfei. Research on Mechanism and Law of EHD Microscale 3D Printing Injection [J]. Journal of Agricultural Machinery, 2016,47(06):401-407.

[17] Yao Guangmin, Wei Bin, Ren Wucheng, et al. Numerical Simulation of Particles Collected by Charged Drops in Shower Room [J]. Energy Saving, 2015, 34(11):41-44.

[18] S. Humphries, Charged Particle Beams, Dover Publications, New York, 2013.

[19] Yuan Yin, Wang Jinggang. Numerical Simulation of Particle Motion Trajectory in Charged Water Mist Dedusting Process [J]. Journal of Beijing University of Chemical Technology (Natural Science Edition), 2005, 32(1). 